\title{
La experiencia docente del Observatorio de derechos humanos en la formación de juristas comprometidos
}

José Elías Esteve Moltóa, Raquel Vanyó Vicedob, Joan-Marc Ferrando Hernández ${ }^{\mathfrak{c}}$ y Estrella del Valle Calzada $^{\mathrm{d}}$

aProfesor Titular de Derecho Internacional y Relaciones Internacionales, Universitat de València (IDHUV), ${ }^{b}$ Profesora Contratada Doctora de Derecho Internacional y Relaciones Internacionales, Universitat de València (IDHUV), 'Investigador predoctoral de Derecho Internacional y Relaciones Internacionales, Universitat de València (IDHUV) y ${ }^{d}$ Investigadora predoctoral de Derecho Internacional y Relaciones Internacionales (Programa Atracción de Talento UV) (IDHUV)

Esta actividad se enmarca en el desarrollo del Proyecto de Innovación Docente de la Universitat de València: NOUPID UV-SFPIE_PID20-13523029

\section{\$EWWFW}

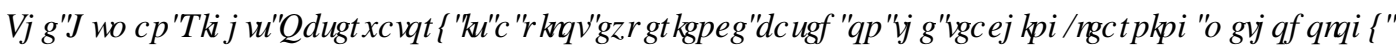

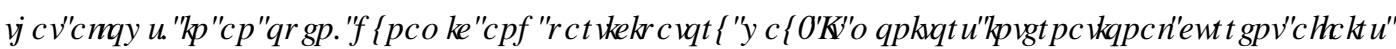

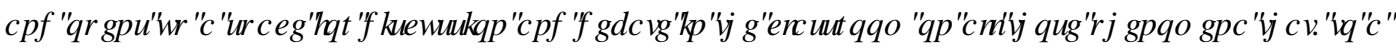

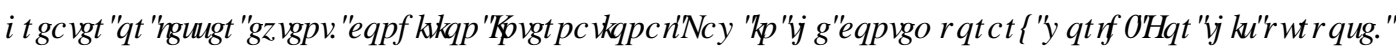

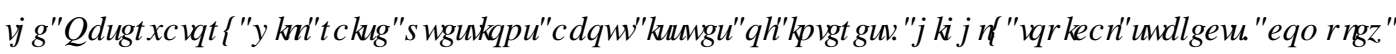

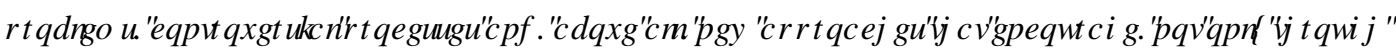

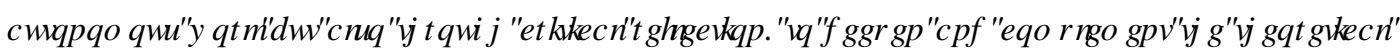

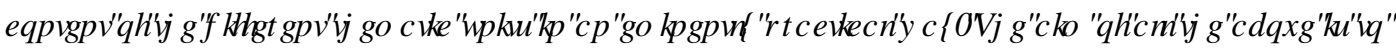

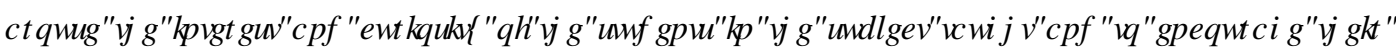

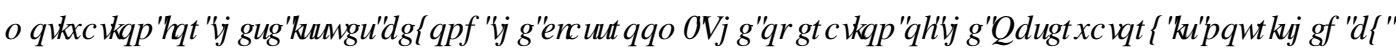

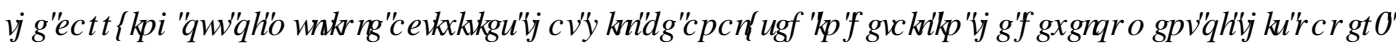

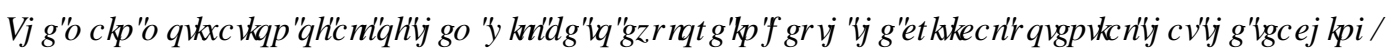

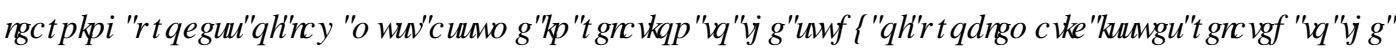
SLRUFWRQDQGISLRP RURQRID+ XP DQ5 IJKWD

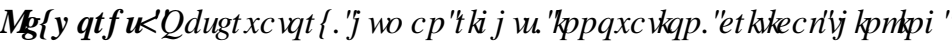

\section{HXP HQ}

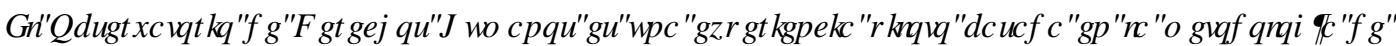

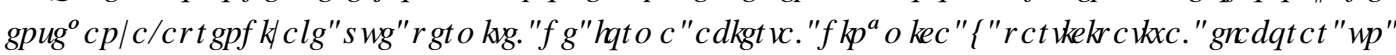

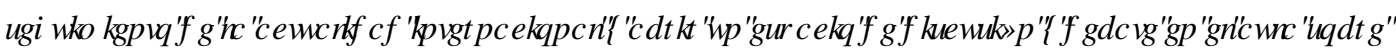

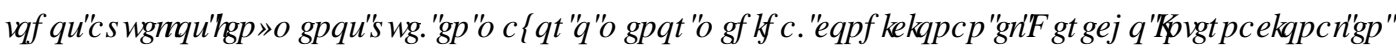

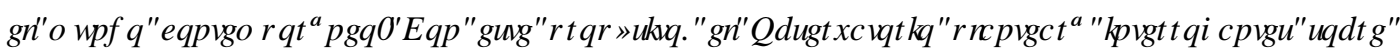

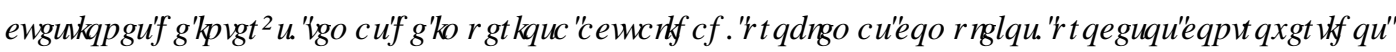

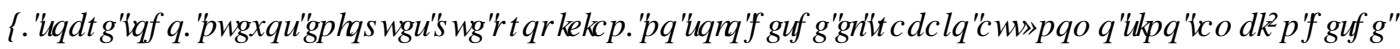

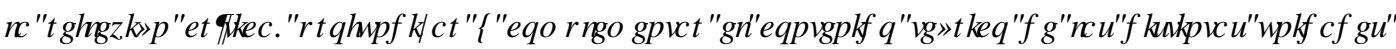

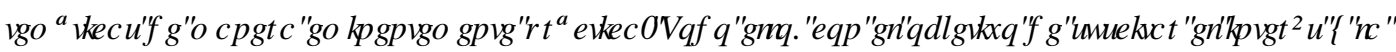

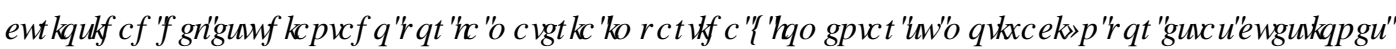

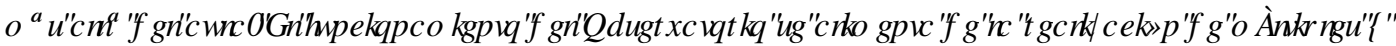




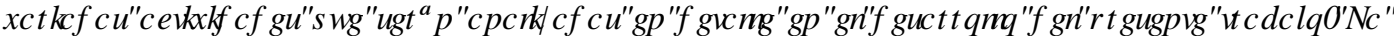

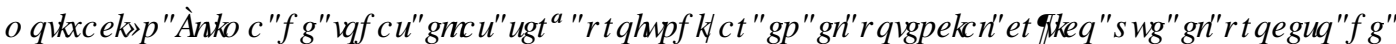 HQMKDQ]DDSUHQ]DNAI GHD ' HHFKR GEH DUP IU HQ UHDFIYQ FRQ HD HMXGR】 GH FXHMRQHD

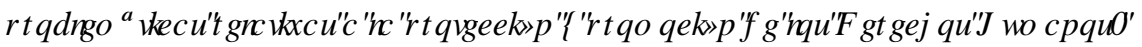

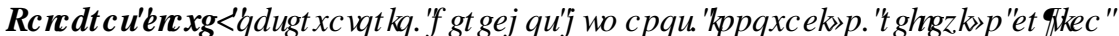

[

\section{Introducción}

El Observatorio de Derechos Humanos es una experiencia piloto basada en la metodología de enseñanzaaprendizaje que permite, de forma abierta, dinámica y participativa, elaborar un seguimiento de la actualidad internacional y abrir un espacio de discusión y debate en el aula sobre todos aquellos fenómenos que, en mayor o menor medida, condicionan el Derecho Internacional y de los Derechos Humanos en el mundo contemporáneo. Esta propuesta se basa en la interiorización de la idea de que, parafraseando al jurista alemán Von Ihering, cualquier derecho en el mundo tiene que ser conseguido mediante lucha.

El Observatorio también responde a la gran responsabilidad que el profesorado de las disciplinas jurídicas debe tener. En este sentido, el proyecto parte de la premisa de que el derecho es una herramienta muy poderosa de transformación social, esencial para la sociedad que lo articula, lo regula, lo consume y lo sufre. Por esta razón, es necesario reivindicar la función pública del derecho y revalorizar la importancia que tiene el derecho público en la formación integral del jurista. En este punto, la formación impartida en los programas de Máster y Doctorado constituye un escenario único para reclamar ese espacio. La finalidad de todo ello no es solamente formar a juristas, sino formar juristas comprometidos con la sociedad en la que vivimos y que los participantes asuman un perfil de vocación pública y de sentido de la justicia que es ahora más necesario que nunca. Así, esta experiencia piloto permite reforzar la formación reflexiva a partir del estudio de los conflictos que generan las violaciones de derechos humanos tanto en el ámbito estatal como internacional, con el objetivo de generar en los estudiantes lo que se conoce como conflicto cognitivo, esto es, mostrar las disparidades entre las formas tradicionales en las que se estudia el derecho y los derechos humanos y las constantes vulneraciones que se producen de estos. Por otro lado, se hace necesario mencionar la inevitable vinculación de esta propuesta al trabajo realizado en el marco de la Clínica Jurídica por la Justicia Social de la Universitat de València. Muchas de las dinámicas y actividades que plantea el Observatorio están directamente relacionadas con la metodología clínica por tratarse de una apuesta integral de formación, enseñanza e investigación que lucha por la igualdad y la justicia social.

El Observatorio de Derechos Humanos se caracteriza por su retroalimentación ya que se nutre de la actualidad del panorama internacional y de las temáticas que, año tras año, sean trascendentales en el plano internacional. Una idea fundamental es la escogida como línea temática anual que ocupará al Observatorio y que será objeto de seguimiento exhaustivo durante todo el curso académico. De manera paralela a esta línea central, se plantea la creación de una segunda línea que abarque el seguimiento permanente de la actualidad internacional con el foco puesto en aquellos asuntos que vayan surgiendo en el plano 
internacional. No obstante, debe señalarse que de igual forma que cambian los temas de interés y actualidad internacional, se adaptan las actividades a realizar, por lo que resulta impracticable el desarrollo de un listado exhaustivo de actividades concretas. Ahora bien, las opciones son numerosas: desde la realización de ciclos de seminarios, el establecimiento de mecanismos de seguimiento de la actualidad en términos de Derechos Humanos, Derecho Internacional y Relaciones Internacionales, así como la creación de material de divulgación y denuncia de vulneraciones de derechos humanos. Por medio de este tipo de actividades, se pretende profundizar en el potencial crítico que el proceso de enseñanza-aprendizaje del derecho debe asumir en relación con el estudio de cuestiones problemáticas relativas a la protección y promoción de los Derechos Humanos.

Esta iniciativa está siendo implementada en la actualidad en los Programas de Máster y Doctorado en Derechos Humanos, Democracia y Justicia Internacional de la Universitat de València, como parte de un Proyecto de Innovación Docente concedido por la propia Universidad. Por las características que definen su estructura, este proyecto es plenamente apto para ser adaptado a las exigencias de la docencia virtual que los tiempos presentes imponen, así como también, en su caso, ser desarrollado en su modalidad híbrida o íntegramente presencial, en atención a las circunstancias.

El Observatorio, pues, se plantea como una herramienta de formación integral para futuros juristas que trata de trascender del ámbito acotado del aula. De hecho, está pensado no solo como un espacio abierto a la reflexión y al diálogo sino a la cultura en general. Es por eso que dentro del dinamismo del Observatorio se encuentra como una actividad fundamental la invitación de especialistas, expertos o víctimas de vulneraciones de derechos humanos para que expongan sus investigaciones o situaciones personales para enriquecer el proceso de formación del alumnado como una ciudadanía crítica, comprometida con la sociedad y con los derechos humanos.

\section{Objetivos principales}

La formación de juristas comprometidos con la sociedad en la que viven debe comenzar necesariamente en las aulas de la Facultad. Para lograrlo, el Observatorio de Derechos Humanos integra cuatro perspectivas diferentes pero interrelacionadas: la perspectiva crítica, la perspectiva de derechos humanos, la perspectiva de género y la perspectiva interdisciplinaria.

En primer lugar, la incorporación de la perspectiva crítica en la metodología del proyecto aspira a que el alumnado adquiera competencias relativas al razonamiento crítico a través del debate y la discusión de argumentos, de manera que así mejore su capacidad analítica de la información y de la normativa. En segundo lugar, el Observatorio pretende detenerse en el estudio específico de los problemas que suscitan mayor controversia en la esfera internacional y analizarlos desde el punto de vista de su incidencia en los derechos humanos. En tercer lugar, el análisis de cualquier tema que se trate en el Observatorio debe asumir inexcusablemente la perspectiva de género, puesto que este enfoque representa un elemento transversal, 


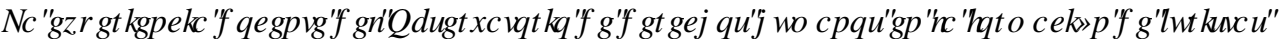 FRP SLRP HMGRV}

que debe orientar la mirada crítica y de derechos humanos sobre las materias abordadas. El Observatorio, como espacio de reflexión crítica, procura reforzar el compromiso con la igualdad de género y dar la adecuada visibilidad a todos aquellos aspectos que afectan a las mujeres. En cuarto y último lugar, el Observatorio de Derechos Humanos integra una perspectiva interdisciplinar en su metodología de trabajo para alcanzar sus objetivos. La realidad es compleja, por lo que resulta imprescindible el análisis de conjunto. En el plano internacional los fenómenos se entrecruzan, están interconectados y cambian en función de múltiples factores. Por esta razón, el Observatorio apuesta por una visión holística que incluya otras disciplinas más allá del Derecho, tales como las Relaciones Internacionales o la Sociología, etc. para lograr una mejor comprensión del funcionamiento de la sociedad en la que vivimos.

A la vista de lo anteriormente explicado, el Observatorio de Derechos Humanos, como propuesta de innovación docente, se propone alcanzar diversos objetivos específicos de carácter académico, competencial y social, de acuerdo con las perspectivas mencionadas. En particular, el Observatorio aspira a ofrecer una visión práctica de las asignaturas con las que se relaciona, sin perder de vista los principales problemas que se plantean para el Derecho Internacional y los Derechos Humanos. El ámbito de aplicación de esta propuesta docente abarca, como se ha mencionado anteriormente, desde los Grados universitarios hasta los estudios de Máster y el Programa de Doctorado. Aunque los objetivos sirven para todo el elenco de formación universitaria, el nivel de profundidad y complejidad puede modularse para adaptarlos al grupo.

Asimismo, esta propuesta de innovación docente quiere desarrollar la capacidad analítica del estudiantado a través del debate en el aula y del trabajo individual y grupal, también con el objetivo de mejorar las competencias de redacción y oralidad, mostrando especial atención a la argumentación jurídica para resolver problemas complejos. Este objetivo pasa por la promoción de la interacción y la comunicación de manera presencial pero también por vía de herramientas virtuales, habida cuenta de la situación sanitaria imperante. Ello enlaza con otro de los objetivos generales de la propuesta de innovación docente como es que el Observatorio aspira a trascender el espacio del aula. Como corolario de estos objetivos, el Observatorio apuesta poderosamente por la formación integral de los y las juristas con vocación de justicia y compromiso social.

\section{Metodología, desarrollo de la innovación y evaluación de las actividades}

Este proyecto de innovación pretende generar un impacto directo en lo que ha de ser considerado un elemento esencial en la metodología universitaria de enseñanza, como es la formación de los y las estudiantes en competencias y valores para conseguir una sociedad comprometida con los derechos humanos y con el cumplimiento del derecho.

En este sentido, el cumplimiento de los objetivos específicos propuestos fomentará que el alumnado se aproxime a una visión de carácter práctico de la realidad internacional. De esta manera, el proceso de 
aprendizaje se enriquecerá con la aportación de espacios aptos para la crítica, el debate e incluso, en caso de que así se estimara necesario, la denuncia pública. Todo esto, como resultado de un proceso de profundo estudio y minucioso análisis de los hechos que acontecen en la sociedad global e interconexionada en la que vivimos, en un proceso de aplicación práctica de los conocimientos legales teóricos impartidos en las sesiones.

Con el fin de alcanzar los objetivos anteriormente descritos, el Observatorio de Derechos Humanos plantea toda una serie de actividades de diversa índole que se desarrollan en consonancia con los presupuestos pedagógicos y de innovación docente que exige el Espacio Europeo de Enseñanza Superior (EEES).

\subsection{Metodología empleada}

El Observatorio diseñado, aunque se trata de una experiencia piloto de innovación educativa, no deja de lado la metodología clásica de las ciencias jurídicas. Se recurre también a estos métodos de enseñanza magistral, que consideramos igualmente necesarios, aunque complementándolos siempre con materiales interactivos, tales como vídeos y documentales, para trabajar sobre cuestiones diversas. De este modo, tratamos de adecuar el entorno en que se desarrolla el proceso de enseñanza-aprendizaje a las nuevas herramientas que permiten un desarrollo de las metodologías docentes desde un enfoque innovador y ajustado a las nuevas realidades y herramientas de las que disponemos.

Es en esta miscelánea de técnicas educativas o metodológicas donde radica una de las razones que aportan originalidad a esta propuesta; hablamos de la combinación de la metodología clásica para la docencia jurídica, con, entre otros, metodologías de enseñanza-aprendizaje, aula inversa y metodología basada en la adquisición de competencias.

A este respecto, resulta particularmente relevante el enfoque de la enseñanza basada en la adquisición de competencias profesionales y de pensamiento y reflexión orientadas hacia la formación de una ciudadanía comprometida. Así, encontramos en el aula un lugar idóneo para no solo transmitir conocimientos puramente teóricos del Derecho Internacional de los Derechos Humanos, sino para orientarlos hacia la adquisición de competencias específicas por parte del alumnado, más allá de la mera asunción de los contenidos. En consecuencia, las diversas actividades y dinámicas que plantea el Observatorio a lo largo de cada uno de los cursos académicos en los que se implementa tienen como finalidad última dar cumplimiento a los presupuestos competenciales que tendrán que demostrar los futuros profesionales en nuestra sociedad una vez acabados sus estudios universitarios.

Otro elemento que proporciona originalidad a la propuesta radica en la variedad de actividades que la componen, de entre las que podemos encontrar seminarios, cinefórums, publicación de artículos en blogs, talleres formativos... Todo ello, con el valor añadido de que se producirá un flujo constante de creación de contenido, vinculado a la actualidad internacional y obtenido como resultado de un profuso estudio y 
análisis procedente de conocimientos de carácter teórico orientados a la práctica de nuestra sociedad y realidad internacional.

\subsection{Desarrollo del Observatorio}

Una de las características que definen al Observatorio es su adaptación y permeabilidad a los hechos que acontezcan en el panorama internacional. En base a ello, las actividades que se realicen en el marco de esta actividad tendrán que ser adaptadas a las circunstancias de cada curso académico, modificándose año tras año.

Con carácter anual, se escogerá una línea temática que será objeto de seguimiento exhaustivo durante el curso académico. Esta temática podrá referirse a la situación de los derechos humanos en un país concreto, un colectivo vulnerable específico o un contexto necesitado de análisis, como la actual relación entre los derechos humanos y los impactos que está generando el Covid-19 en todo el mundo.

Pese a la elección de una línea temática general, ello no obstará para que se sigan analizando otros asuntos de forma simultánea cuya definición se irá adaptando a la actualidad internacional.

Destacamos a modo de ejemplo algunas de las actividades incorporadas al cronograma del presente curso académico 2020-2021, de aplicación a los Programas de Máster y Doctorado en Derechos Humanos, Democracia y Justicia Internacional:

- Ciclo de seminarios virtuales para profundizar de manera específica en un único tema de actualidad en derechos humanos, como pueda ser el análisis de la situación de derechos humanos en un país (2020-2021: Guinea Ecuatorial).

- Programas de cinefórum, con la proyección de películas y documentales vinculados al Derecho Internacional de los Derechos Humanos, así como con la realización de un posterior coloquio

- Creación de material para la divulgación y denuncia de vulneraciones de derechos humanos

- Mecanismos de reporte de la actualidad en términos de derechos humanos, Derecho Internacional y geopolítica, mediante la publicación en diarios informativos y blogs de opinión

- Elaboración de informes sobre ciertos aspectos de derechos humanos o sobre las vulneraciones de los mismos que luego puedan servir de base para la presentación de denuncias ante comités de Naciones Unidas

- Formación de tribunales simulados para la formación del alumnado en la experiencia de análisis y desarrollo de un caso judicial por vulneración de derechos fundamentales

- Simulación de procesos de adopción de medidas legislativas, tales como el Congreso de los Diputados (en su reforma del mecanismo de jurisdicción universal), Tribunal Permanente de los Pueblos (como ejemplo de tribunal ético internacional no gubernamental), o entre otros, el Consejo 
de Derechos Humanos (en la negociación del Tratado Vinculante para Empresas y Derechos Humanos).

- Colaboración con órganos judiciales internacionales por medio de la elaboración de DP IFXVFXUDH o con organizaciones no gubernamentales en la redacción de informes técnicos

\subsection{Incorporación de la evaluación a las actividades realizadas}

Dentro del currículo docente, el Observatorio está planteado como actividad que integra la evaluación continuada. A este respecto, se desarrolla en paralelo con las clases teóricas del Programa de Máster y las Actividades de Formación Específica de Doctorado, tratando siempre de mantener una conexión temática coordinada con el contenido teórico impartido en estas: actividades relacionadas con la teoría, pero de carácter eminentemente práctico.

Pese a que curricularmente esta actividad debe insertarse en el marco evaluador de nuestras guías docentes, debemos seguir teniendo en consideración que el proceso de evaluación del Observatorio tratará siempre de ir más allá de la concreción de una mera calificación numérica final. A este efecto, se tienen en cuenta toda una serie de datos que permiten configurar un sistema valorativo múltiple que se ajusta también a los nuevos postulados metodológicos que marca el Espacio Europeo de Educación Superior. En la evaluación de la asignatura, por lo tanto, confluyen todo tipo de métodos de evaluación: desde la evaluación clásica a la coevaluación, donde son los propios estudiantes los que se evalúan entre ellos siguiendo una rúbrica previamente diseñada por el profesorado, o por el profesorado y el estudiantado de forma conjunta.

Entrando en un mayor nivel de detalle, algunas de las propuestas realizadas como formas de evaluación y de demostración de resultados alcanzados son las siguientes:

- Presentación de un informe anual sobre el contenido y valoración reflexiva de los seminarios temáticos realizados, acentuando las conclusiones alcanzadas y las medidas de acción que pudieran ser llevadas a cabo

- Redacción y valoración de artículos y publicaciones sobre las cuestiones analizadas

- Procesos de coevaluación entre el alumnado complementados por la valoración del profesorado, con rúbricas precisas y ajustadas a las actividades realizadas y a las competencias proyectada

\section{Resultados obtenidos}

Pese a que se trata del primer año de implementación del Observatorio, ya han sido múltiples las actividades desarrolladas en el marco del proyecto. A este respecto, y a meros efectos ejemplificativos, hemos destacado algunas de ellas que entendemos que abarcan una pequeña representación de su variedad y 
multidisciplinariedad: seminario de formación específico, cinefórum, taller de formación y actividad vinculada a la línea temática anual escogida.

Por lo que respecta al seminario de formación específico (cartel en Fig. 1), se optó por impartir una sesión basada en la reflexión de un ensayo trabajado con los estudiantes con motivo del Día Internacional de la Mujer. En concreto, la obra escogida fue "Manifiesto de un feminismo para el 99 \%" y la sesión de debate fue dirigida por la Profesora Ruth Mestre, integrante del cuerpo docente del Máster en Derechos Humanos, Democracia y Justicia Internacional de la Universitat de València.

Dentro de la experiencia del Observatorio, también tuvo lugar una sesión de cinefórum en la que se proyectó el documental "Irioweniasi: El hilo de la luna" (cartel en Fig. 2). Este documental, con una duración aproximada de una hora, versa sobre la experiencia migratoria de una joven nigeriana que se ve arrastrada a una red de trata de personas con fines de explotación sexual en Europa. Encabezando el debate realizado tras la proyección del documental, pudimos contar con la presencia de sus directoras, Inmaculada Antolínez Domínguez y Esperanza Jorge Barbuzano, así como con renombradas juristas especialistas en la materia como Tania García Sedano o Maite Parejo. Al hilo de la proyección y de las intervenciones, se abrió un enriquecedor coloquio entre el público y las ponentes, con las que los asistentes pudieron compartir los puntos más controvertidos, tanto a nivel legal como técnico.

En relación con las actividades de formación teórico-práctica, se han impartido hasta la fecha dos talleres. El primero de ellos se trató de un taller para los y las estudiantes orientado a su aproximación a la técnica del litigio estratégico, herramienta jurídica que, en su conceptualización, encaja de forma íntegra con los principios y objetivos proyectados por el Observatorio. Para ello, contamos con la participación de Joaquín Mejía, quien centró su exposición en el contexto territorial de América Latina y narró al estudiantado sus experiencias profesionales en la litigación (cartel en Fig. 3). En segundo término, fue también organizado un taller de formación en colaboración con la organización CEPAIM, cuyo objetivo principal era convertir a los y las asistentes en "agentes antirumores" contra el racismo, la xenofobia y promover la convivencia intercultural (cartel en Fig. 4)

Finalmente, destacamos también a estos efectos la realización de una de las actividades que se enmarca en la línea temática escogida para el curso académico 2020-2021, cual es la situación de los derechos humanos en Guinea Ecuatorial. Para contextualizar el panorama, Mocache Massoko, Director del Diario Rombe, impartió una conferencia con el título "Corrupción y violaciones de derechos humanos en Guinea Ecuatorial" (cartel en Fig. 5). Los y las estudiantes tuvieron la oportunidad de iniciar un enriquecedor diálogo con el ponente, quien les expuso la situación que se está viviendo en el país desde la propia experiencia personal. Este diálogo propició el inicio de una colaboración entre los asistentes y el ponente de cara a la realización de futuras acciones en defensa de los derechos de los ciudadanos ecuatoguineanos.

En la segunda de estas sesiones temáticas, pudimos contar con la participación de Manuel Ollé Sesé, profesor de derecho penal y abogado en ejercicio, que intervino como letrado en una causa relativa a los 
hechos denunciados contra miembros del gobierno de Guinea Ecuatorial (cartel en Fig. 6). Gracias a sus aportaciones, los y las estudiantes que integran el Observatorio pudieron dar continuidad a su análisis de la cuestión temática escogida.

Lo aquí señalado conforma tan solo de una pequeña muestra de las actividades realizadas y de los resultados obtenidos en los primeros meses de andadura de un proyecto de innovación que sigue en marcha y que continuará su desarrollo en los próximos cursos académicos de las titulaciones mencionadas.

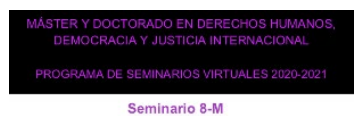

Seminario 8-M

Explorando el feminismo para el $99 \%$

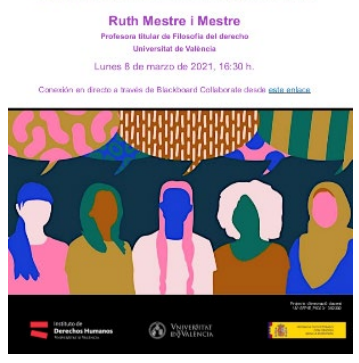

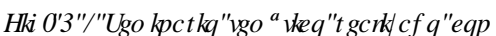

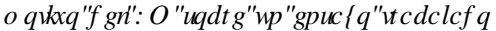
FRQ HD DOPQDAR] ${ }^{3} 0$ DQIIHMK SDW XC IH IQLP RISDWHO II'

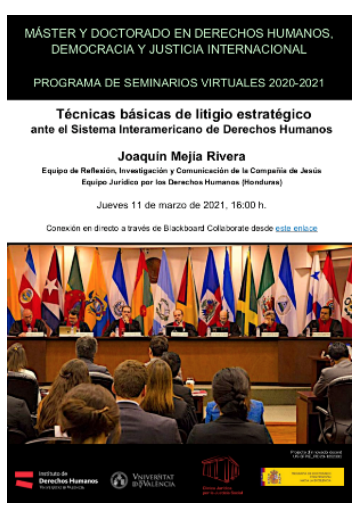

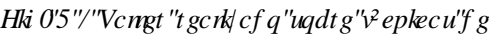
QWIR] HWDWJIFR] SDW (D) IRLPDLC SU FUFR WHUFDCHODOP QDAR]
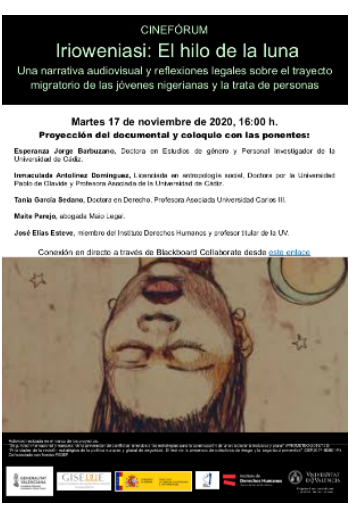

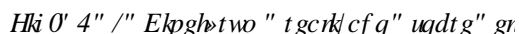
GRFXP HQWD, URZ HQIDMD FRQD DSUMHFWHC

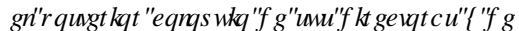
HSHIDOWDWHQDPP DUHD

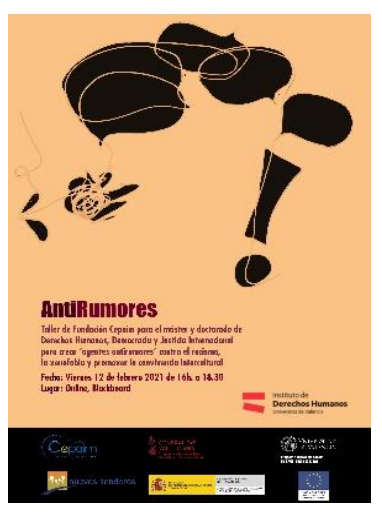

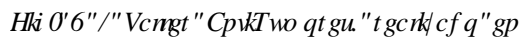

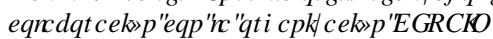




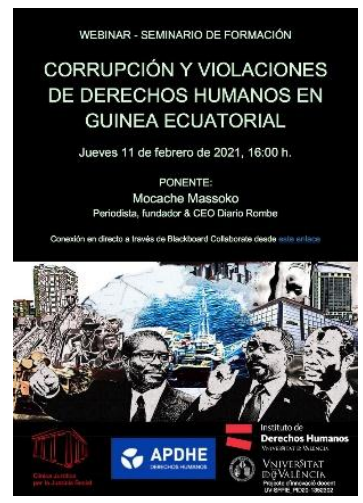

) $I J \square \square[3$ UP HRTGHCRVUP LQDURVUHDQ]DQRI VREH(D)QQHDUP $\mathrm{U}$ WFDHFRJIODHQHDFXUR

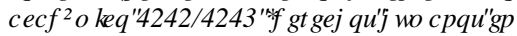
* XIQHDI FXDIRUDO

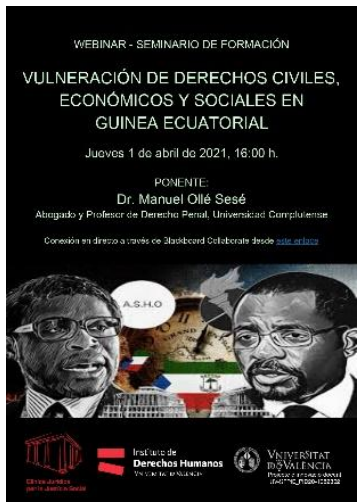

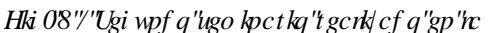
$P H Q F R Q D D$ QQQHD UP $\mathrm{WFD}$ FRQ Q SDUAFISDFIYQ GHD DERJDOR] 0 DQXHD 2 QD $6 H \mathrm{p}$

\section{Conclusiones}

Si el objetivo de las Facultades de Derecho es la formación de juristas, esta formación ha de encaminarse necesariamente a la adquisición de su compromiso real con la sociedad. Es hacia la consecución de este objetivo donde podemos ubicar la implantación del Observatorio de Derechos Humanos como una herramienta de formación integral que pretenda trascender del ámbito acotado del aula.

Esta labor pretende ser abordada por el Observatorio por medio de la integración de cuatro perspectivas interrelacionadas, como son la perspectiva crítica, la perspectiva de derechos humanos, la perspectiva de género y la perspectiva interdisciplinar. Estos enfoques, a su vez, se ven materializados por la realización de múltiples actividades, seminarios, talleres o formaciones, en las que los y las estudiantes trabajarán en las competencias que deben adquirir, tanto como futuros profesionales, como sobre todo, como futuros juristas comprometidos con los problemas de su entorno.

La percepción de la utilidad de ciertas áreas del derecho o conocimientos cambia drásticamente cuando los estudiantes descubren que sus tareas de investigación y análisis tienen un impacto real (y trascienden de la mera evaluación). En estos casos, tal y como sugiere la experiencia extraída con este proyecto, la implicación con las causas de derechos humanos por parte del estudiantado aumenta.

En este sentido, aunque este proyecto piloto está siendo implementado en programas académicos vinculados al Derecho Internacional y al Derecho Internacional de los Derechos Humanos, a la vista del impacto generado en el estudiantado, tanto en su proceso de aprendizaje como en su compromiso con los temas analizados, entendemos que la experiencia podría ser trasladada con éxito a otras ramas del derecho o incluso a otras disciplinas de las ciencias sociales. 
Con todo, y a modo de conclusión, el proyecto del Observatorio de Derechos Humanos aspira a conseguir, por medio de esta metodología de enseñanza-aprendizaje, la formación de juristas comprometidos con la sociedad en la que vivimos. Es en estos momentos de crisis, como la generada por la actual pandemia, cuando son más necesarias que nunca las conciencias críticas. Tal vez así, podamos contribuir a la construcción de sociedades más justas.

\section{Referencias}

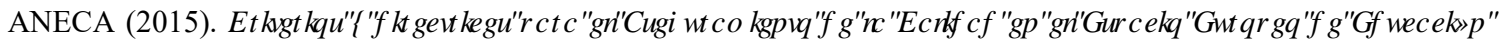
6XSHIRU/ $(6 *$ A Aprobado por la Conferencia de Ministros celebrada en Ereván el 14 y el 15 de mayo de 2015.

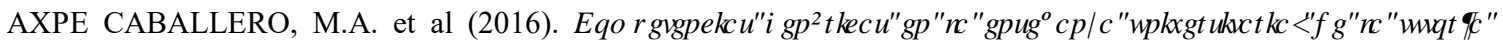
IRLP DUYDDLDIQUAJ UFIy QFXUFXФUMMálaga: Aljibe.

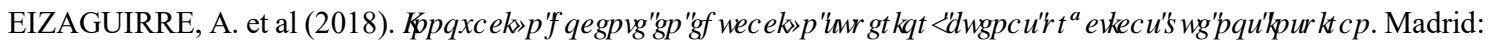
Pearson.

España: Ley Orgánica 4/2007, de 12 de abril, por la que se modifica la Ley Orgánica 6/2001, de 21 de diciembre de Universidades (BOE, núm. 89, de 13 de abril de 2007).

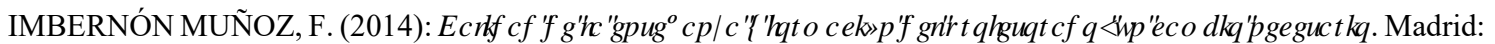
Octaedro.

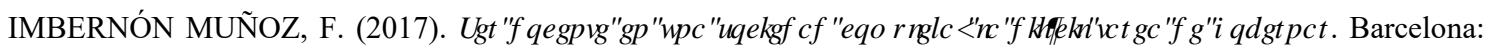
Graó.

IMBERNÓN MUÑOZ, F., y GUERRERO ROMERA, C. (2017). “¿Existe en la universidad una profesionalización docente?" en 5 HLWDOGH( GXFDFIy QDD' LWDQFDDI56 (11).

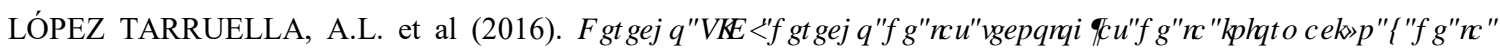
FRP XQIFDFly QIValencia: Tirant lo Blanch.

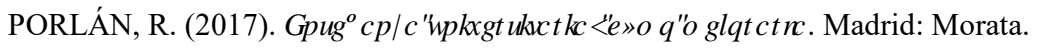

